\title{
All things must pass
}

Shaun Sellars continues this series on ethical dilemmas in dentistry which appears in every second issue of the $B D$ J.

The GDC recently announced that Bill Moyes, Chair of the council, is to stand down from his role in 2021. With this, the GDC has an opportunity to move on from a period where it has received criticism from both the profession it regulates and the Professional Standards Authority (PSA) who oversees the GDC itself.

Many will remember the Moyes era of the GDC for the rebranding of our patients as consumers. In his Pendlebury Lecture of 2014, Dr Moyes proclaimed that 'dentists and dental care professionals now have customers, not clients...or, indeed, patients.' Fortunately, most of us still consider ourselves healthcare professionals dealing with patients, rather than salespeople.

Unsurprisingly, there has been much speculation about a successor to Dr Moyes, with a vocal section of the profession insisting that the new Chair is a registrant.

On the face of it, this seems to be a sensible way forward. Who better to help direct the regulation of the profession than an insider who understands the specific issues that dentists and DCPs face, especially given the perception of recent overregulation and fear of fitness to practise investigations?

Having a registrant as Chair may raise a few issues, least of all being who would take the role. Apart from it being a bit of a poisoned chalice, partly thanks to the vilification of the departing Chair, the specific qualities required for the position means that the number of eligible applicants is limited. And this also ignores the potential for reduced transparency and independence that comes with having a registrant in charge.

But is there another possibility? Having an experienced layperson as Chair of the regulatory council is an option which many of the professions governed by the PSA have chosen.

Again there are advantages and drawbacks of this. A layperson, by definition, has no direct experience of the profession they're regulating. A robust supportive council, where the registrant members feed into the Chair on their areas of expertise, can help mitigate this.

Let's not throw the baby out with the bathwater, though. Having a non-registrant

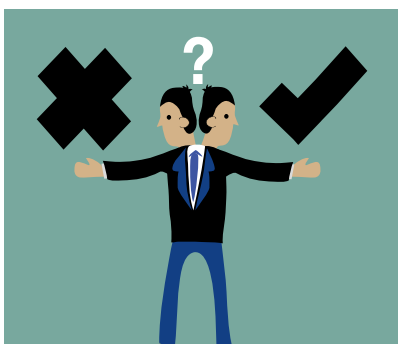

Chair may have benefits. The obvious benefit is that a non-registrant is, theoretically at least, able to bring knowledge from out of the profession and integrate it into dentistry. Dr Moyes himself did this, albeit to the detriment of dentistry and perception of the GDC. But imagine, for example, a Chair who brings with them knowledge of the airline industry and their 'no blame' culture of error reporting. The effect this could have on the fitness to practise process would be profound, and the knock-on effect onto dentistry would be immense.

The GDC now face a choice. Finding a Chair who can regain the lost trust and respect of the profession as well as altering the course of dental regulation for the better is going to be tough. Whatever their choice, the number one desirable factor is an understanding of regulation, preferably with a healthcare background and an insight into how dental regulation has failed over recent years.

\section{Revised guidance on radiation protection published}

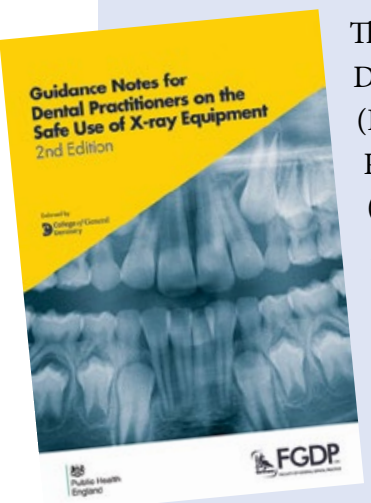

The Faculty of General Dental Practice (FGDP $[\mathrm{UK}]$ ) and Public Health England (PHE) have published revised guidance on radiation protection in dental practice.

The second edition of Guidance Notes for Dental Practitioners on the Safe use of

$X$-ray Equipment (https://bit. ly/35DYFb7) sets standards of good practice for the safe use of X-ray equipment in dental practice, and is endorsed by the College of General Dentistry (CGDent).

The guidance is freely available on the
FGDP website, where hard copies are also available to purchase from the online shop, and FGDP members based in the UK will shortly be receiving a complimentary hard copy.

Presenting definitive, detailed and comprehensive recommendations in a logically ordered and intelligible format, the guidance notes are designed as a reference for the whole primary care dental team. They cover both administrative and practical matters, taking into account developments in dental X-ray equipment, imaging technology and legislation since the first edition, which was published in 2001 by the National Radiological Protection Board.

It was produced by a working party led by Public Health England and consisting of regulatory bodies, professional bodies representing dentistry and radiation protection, consultant dental radiologists and general dental practitioners.

The guidance supports compliance with the Ionising Radiations Regulations 2017 (IRR17) and the Ionising Radiation (Medical Exposure) Regulations 2017 (IRMER17), but does not impose any requirements on employers beyond those required by legislation. Ian Mills, Dean of FGDP(UK) and Trustee of the College of General Dentistry, said: 'For almost two decades, this has been an important text in setting standards for the safe use of X-ray equipment in dental practices, and this updated edition will undoubtedly continue to be a key reference document for the whole primary care dental team' 